\title{
Biological sex influences susceptibility to Acinetobacter baumannii pneumonia in mice
}

\author{
Sílvia Pires, ${ }^{1}$ Adeline Peignier, ${ }^{1}$ Jeremy Seto, ${ }^{2}$ Davida S. Smyth, ${ }^{3}$ and Dane Parker ${ }^{1}$ \\ 'Department of Pathology, Immunology and Laboratory Medicine, Center for Immunity and Inflammation, Rutgers New \\ Jersey Medical School, Newark, New Jersey, USA. ${ }^{2}$ Department of Biological Sciences, New York City College of Technology, \\ Brooklyn, New York, New York, USA. ${ }^{3}$ Department of Natural Sciences, Eugene Lang College of Liberal Arts at The New \\ School, New York, New York, USA.
}

Acinetobacter baumannii ( $A$. baumannii) is an extremely versatile multidrug-resistant pathogen with a very high mortality rate; therefore, it has become crucial to understand the host response during its infection. Given the importance of mice for modeling infection and their role in preclinical drug development, equal emphasis should be placed on the use of both sexes. Through our studies using a murine model of acute pneumonia with $A$. baumannii, we observed that female mice were more susceptible to infection. Likewise, treatment of male mice with estradiol increased their susceptibility to infection. Analysis of the airway compartment revealed enhanced inflammation and reduced neutrophil and alveolar macrophage numbers compared with male mice. Depletion of either neutrophils or alveolar macrophages was important for bacterial clearance; however, depletion of alveolar macrophages further exacerbated female susceptibility because of severe alterations in metabolic homeostasis. Our data highlight the importance of using both sexes when assessing host immune pathways.

Conflict of interest: The authors have declared that no conflict of interest exists.

Copyright: () 2020, American Society for Clinical Investigation.

Submitted: September 25, 2019

Accepted: March 11, 2020

Published: March 19, 2020.

Reference information: JCI Insight. 2020;5(7):e132223.

https://doi.org/10.1172/jci.

insight.132223.

\section{Introduction}

Acinetobacter baumannii (A. baumannii) is an emerging opportunistic and extremely versatile multidrug-resistant pathogen that has become a serious global threat, resulting in high mortality rates $(1,2)$. Its extreme genome plasticity and ability to quickly develop resistance to a plethora of antibiotics makes it an important pathogen and one of the reasons it has been designated as a serious threat and critical target by the CDC and World Health Organization (3-7). This has driven significant research in recent years to understand how the host senses and responds to $A$. baumannii infection $(8,9)$. However, given that the emergence of $A$. baumannil is relatively recent, there are still several aspects of the immune response that have not been fully investigated.

Differences in susceptibility between females and males have been observed in the contexts of vaccination, autoimmune diseases, and infectious diseases (10-13). However, sex-based differences and the host immune mechanisms behind this differential response have only recently begun to be dissected. Several studies have presented data showing differential immune response to infectious diseases according to sex (14-16) The innate immune response of females is typically stronger in the context of infection (12). Here we report that female mice have increased susceptibility to A. baumannii-induced pneumonia, and we demonstrate that this difference is due to differential innate immune responses. Alveolar macrophages were found to play a more substantial role in clearance of $A$. baumannii from female mice compared with males. The exacerbation of this sex difference through alveolar macrophage depletion was found to be due to severe mitochondrial alterations that resulted in a metabolic imbalance, leading to a defective response to the pathogen. Our data highlight the importance of using both sexes when assessing host immune pathways to $A$. baumannii and point to a crucial metabolic role for alveolar macrophages in controlling infection in female mice.

\section{Results}

Female mice are more susceptible to A. baumannii-induced pneumonia. While establishing a nonlethal acute pneumonia model with the $A$. baumannii highly virulent clinical isolate 5075, we noticed that female mice had increased susceptibility to infection (Supplemental Figure 1; supplemental material available online with this article; https://doi.org/10.1172/jci.insight.132223DS1). To confirm this observation, weight-matched 
C57BL/6J female and male mice (Figure 1A) were intranasally infected with $A$. baumannii 5075 to account for potential differences in lung volume or upper respiratory tract dimensions. While $88 \%$ of male mice were able to survive, only $12 \%$ of female mice survived $(P<0.001)$ (Figure $1 \mathrm{~B})$. In a nonlethal model of infection, female mice had bacterial counts 939-fold higher $(P<0.0001)$ in bronchoalveolar lavage fluid (BALF), 2076fold higher in lung tissue $(P<0.0001)$, and over 40,000 times greater in the spleen $(P<0.0001)$ compared with male mice 24 hours after infection (Figure $1 C$ ). Consistent with the increased bacterial numbers, female mice had decreased body temperatures $\left(3.9^{\circ} \mathrm{C}\right.$ decrease vs. $0.7^{\circ} \mathrm{C}$, Figure $\left.1 \mathrm{D}, P<0.0001\right)$ compared with male infected mice. Consistent with the poor prognosis, female mice had protein content levels $62 \%$ higher than male mice in their airways $(P<0.05)$, as a sign of lung injury (Figure 1E). Pathological examination of infected lungs from both sexes further confirmed the exacerbated phenotype in females. Male infected lungs showed evidence of inflammatory infiltrates and largely interstitial inflammation (Figure 1F). Microabscesses composed of neutrophils were observed within the lung parenchyma (Supplemental Figure 2A; arrow) while some hemorrhaging and edema were also observed. Lungs of female infected mice showed marked levels of pulmonary edema (Supplemental Figure 2B), hemorrhaging, and multifocal interstitial inflammation with regions of marked consolidation. Multiple lung lobes were involved. There was also evidence of fibrin within airways (Figure 1F; arrow) and neutrophils in airspaces (Supplemental Figure 2C; arrow). The overwhelming increase in bacteria was also evident in female mice, with bacteria readily observed within regions of edema (Supplemental Figure 2D; arrow).

Studies on the innate immune response to $A$. baumannii-induced pneumonia can display conflicting results based on the virulence of the strain utilized $(17,18)$. We repeated our weight-matched studies (Supplemental Figure 3) with a less virulent strain to assess whether the sex difference was strain specific. Consistent with our previous observation, female mice were more susceptible to infection with $A$. baumannii strain ATCC 19606. Bacterial counts were 5-fold higher in BALF $(P<0.001)$ and 19 -fold higher in lungs (Supplemental Figure 3, A and B; $P<0.0001$ ). Female mice infected with ATCC 19606 also had decreased body temperatures compared with male infected mice (Supplemental Figure 1C). No differences in bacterial counts were observed in the spleen between sexes (Supplemental Figure 1B), presumably a result of less extrapulmonary dissemination given its decreased virulence.

Gut microbial differences and dysbiosis have been associated with several lung diseases, including bacterial infections (19-21). To assess whether the gut microbiome was an underlying cause for the increased susceptibility, fecal samples from female and male mice were collected immediately before infection. Fecal microbiome analysis revealed similar beta diversity between the microbiome of female and male mice, leading to a similar clustering of bacterial strains between the sexes (Supplemental Figure 4). This suggests that the gut microbiota is not a key contributing factor to the differences observed in response to A. baumannii lung infection between female and male mice.

Treatment with 17ß-estradiol increases male mice susceptibility to A. baumannii lung infection. To begin dissecting the sex differences, male mice were treated with $17 \beta$-estradiol, the major female sex hormone, during adult life. Male mice treated with estradiol had bacterial counts that were 5.9-fold higher in BALF (Figure 1G; $P=0.057), 2800$-fold higher in lung tissue $(P<0.001$; Figure $1 \mathrm{G})$, and 317-fold higher in the spleen $(P<0.001$; Figure $1 \mathrm{G})$ compared with vehicle controls. Concordant with the increases in bacteria, $17 \beta$-estradiol-treated males had a significant decrease in body temperature compared with their control counterparts $\left(3.3^{\circ} \mathrm{C}\right.$ vs. $1.2^{\circ} \mathrm{C}$, Figure $\left.1 \mathrm{H}\right) . \mathrm{H} \& \mathrm{E}$ staining of the lung revealed increased pathology in the infected males treated with estradiol when compared with ones treated with the vehicle control (Supplemental Figure 5). Lungs of male vehicle-infected mice showed similar pathology as described before, with interstitial inflammation and inflammatory infiltrates across multiple lobes and microabscesses throughout. Males treated with estrogen prior to infection exhibited regions of substantial consolidation and loss of alveolar architecture, dilated vessels, and edema.

Sex steroid hormones have been described to directly influence bacterial growth and metabolism (2224). To test whether estradiol could be exerting a direct effect on $A$. baumannii growth, bacteria were grown in both Luria-Bertani (LB) and M9 minimal media supplemented with glucose, with and without estradiol. In the conditions tested, estradiol did not alter the growth of A. baumannii (Supplemental Figure 6, A and B). Given the importance now placed on utilizing both males and females in research, be it animal or human studies, we further characterized this phenotype by examining the immune responses of these mice.

Female mice display differential immune cell populations and cytokine production in response to respiratory $A$. baumannii infection. It is now well established that sex hormones can directly act and exert an effect on 
A

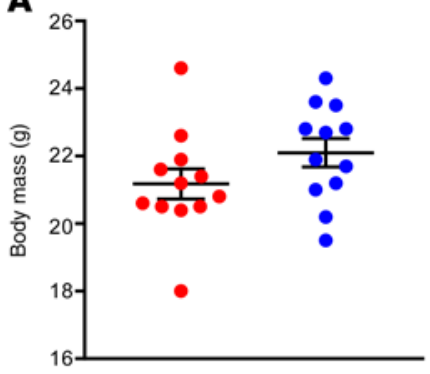

C

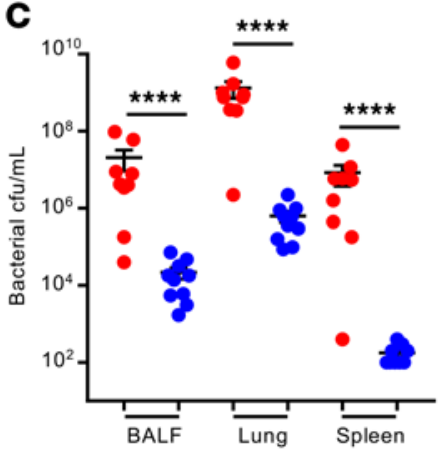

F $\quad$ PBS

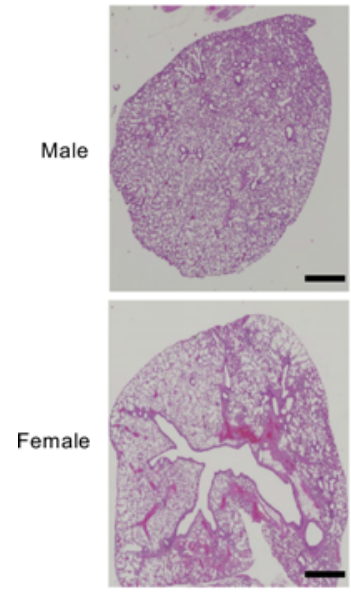

G

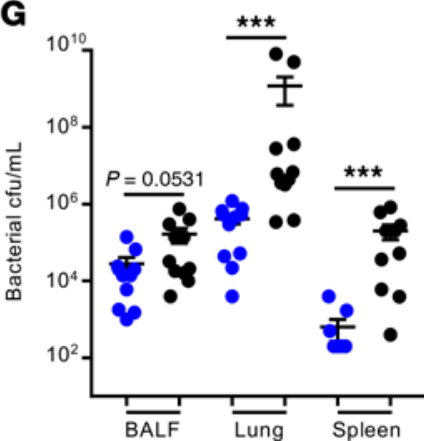

B

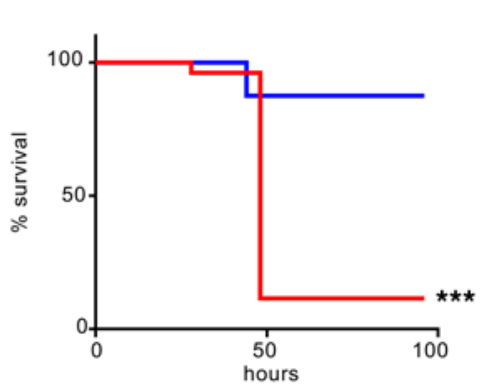

D

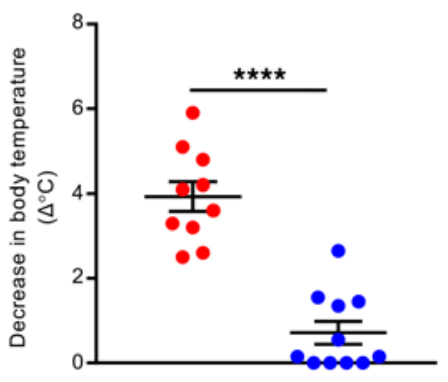

E

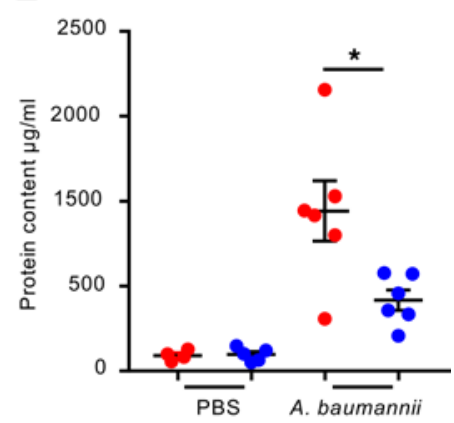

PBS

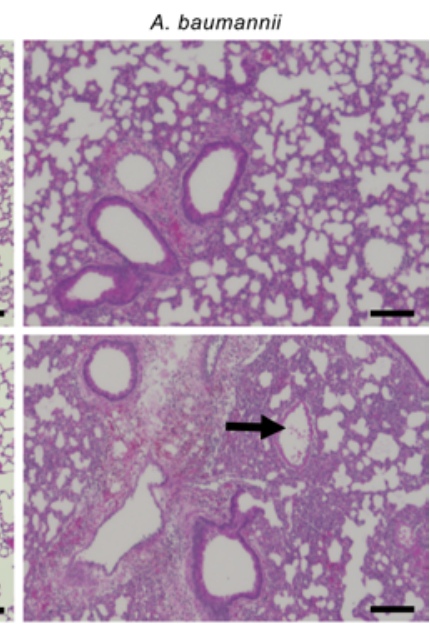

H

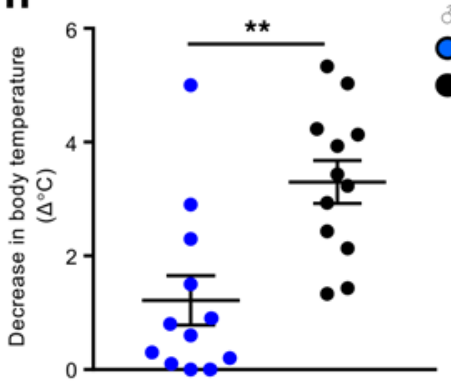

- Female

- Male

- Female

- Male

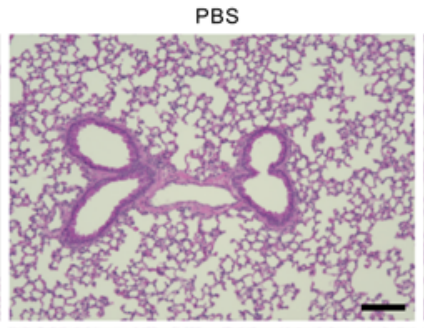

mice

Vehicle

$\beta$-estradiol
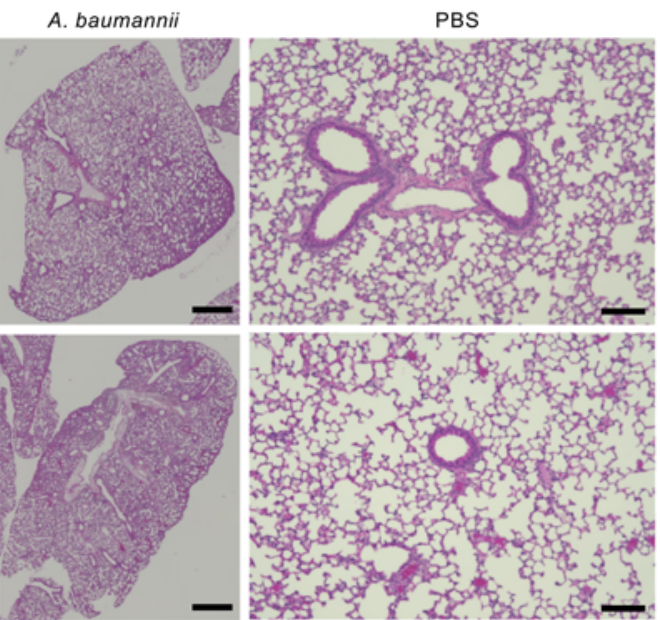

Figure 1. Female mice are more susceptible to pulmonary $\mathbf{A}$. baumannii infection.(A) Weights of mice before infection. (B) Mice were intranasally infected with $10^{8} \mathrm{CFU}$ of $A$. baumannii 5075 and mortality was assessed ( $n=26$ female; $n=8$ male). (C-E) Weight-matched female and male C57BL/6) WT mice were intranasally infected with $10^{7} \mathrm{CFU}$ of $A$. baumannii 5075 for 24 hours $(n=12)$. (C) BALF, lung, and spleen homogenates were enumerated for bacterial counts 24 hours after infection $(n=9)$. (D) Change in external temperature of female and male mice 24 hours after intranasal infection. (E) Protein content in BALF. $n=4$ and $n=5$ for uninfected female and male mice, respectively. (F) Representative H\&E-stained lung sections of mice at 24 hours after infection. Scale bars: $1 \mathrm{~mm}$ (left), $100 \mu \mathrm{m}$ (right). ( $\mathbf{G}$ and $\mathbf{H}$ ) Male mice were treated with $17 \beta$-estradiol or vehicle control for 5 days prior to intranasal infection with $10^{7}$ CFU of $A$. baumannii 5075 ( $n=11$ ). (C) BALF, lung, and spleen homogenates were enumerated for bacterial counts 24 hours after infection. (H) Change in external temperature of mice after infection ( $n$ $=12$. . Each point represents a mouse. Lines display means with SEM. Data are from 3 independent experiments. ${ }^{*} P<0.05,{ }^{* *} P<0.01$, ${ }^{* * *} P<0.001$, and ${ }^{* * * *} P<$ 0.0001 . Mortality data were assessed using Fisher's exact test. A nonparametric Mann-Whitney $U$ test was used to assess differences between groups. 
immune cells $(12,25)$. To determine what may be influencing the enhanced bacterial burden in female over male mice, we analyzed the resident and recruited immune cells into the airway via flow cytometry. Both neutrophils and alveolar macrophages were significantly fewer in female infected mice. Neutrophils were reduced by $64 \%(P<0.01$; Figure $2 \mathrm{~A})$ and alveolar macrophages were reduced by $68 \%(P<0.01$; Figure 2A). This is significant because both neutrophils (26) and alveolar macrophages (27) have been reported to play important roles in the clearance of $A$. baumannii from the airway. More subtle changes were observed with other cell populations (Supplemental Figure 7).

The production of cytokines and chemokines by innate immune cells can also differ between the sexes in response to different stimuli, including bacterial infections $(12,28)$. Analysis of the cytokine and chemokine levels in the airway showed that female mice had significantly increased cytokine production in response to A. baumannii, with the majority of the cytokines quantified being higher in female, compared with male, $A$. baumannii-infected mice (Figure $2 \mathrm{~B}$ and Supplemental Figure 8). Proinflammatory cytokines, such as IL-6 (24-fold; $P<0.0001)$, IL-1B (11.7-fold; $P<0.01$ ), IL-17 (24-fold; $P<0.001$ ), and TNF (2.9-fold; $P<0.0001$ ), were all significantly higher in female infected mice (Figure $2 \mathrm{~B})$. While neutrophil numbers were reduced in the airways of female mice, neutrophil chemokines, such as macrophage inflammatory protein 2 (MIP-2) (18fold higher; $P<0.001)$, KC (25.8-fold higher; $P<0.0001)$, and MIP-1A (3.3-fold higher, $P<0.01)$, were all significantly increased (Figure $2 \mathrm{~B}$ ). These massive cytokine levels are likely a consequence of the higher bacterial burdens in the female mice, while the high bacterial burden is likely related to the reduced immune cell recruitment. In vitro killing assays revealed that male alveolar macrophages had increased capacity to control A. baumannii proliferation while female bone marrow-isolated neutrophils showed a trend toward increased proliferation, when compared with male mice following a 4-hour and 1-hour infection, respectively (Figure $2, \mathrm{C}$ and $\mathrm{D}$ ). This difference was more pronounced in alveolar macrophages (Figure $2 \mathrm{C}, P<0.01$ ), serving as another indication of an important role of these immune cells in the sex-differential response to $A$. baumannii infection. Assessment of immune cell function in vivo showed that neutrophils from infected female mice had a reduced capacity to produce reactive oxygen species (ROS) ( $36 \%$ reduction; $P<0.01$; Figure $2 \mathrm{E}$ ) compared with males, maybe indicative of an inability to fight the infection. We also observed increased cell death (3-fold increase; $P<0.05$; Figure 2E) in neutrophils from female infected versus male infected mice. Last, we examined whether exposure to estrogen had a direct impact on immune cell function in vitro. Treatment of both immortalized bone marrow-derived macrophages (BMMs) and the RAW macrophage cell line with $\beta$-estradiol reduced their capacity to kill $A$. baumannii in an in vitro killing assay (Figure $2, \mathrm{~F}$ and $\mathrm{G}$ ).

Alveolar macrophage depletion exacerbates female susceptibility to A. baumanni-induced pneumonia. Evidence for a role for neutrophils and alveolar macrophages in response to $A$. baumannii has been observed $(26,27,29,30)$. The decreased recruitment of these cells observed in female mice (Figure $2 \mathrm{~A}$ ) is likely to contribute to the increased bacterial burden. We therefore tested the relative contribution of each population in male and female mice.

Neutrophils were depleted using a neutrophil-specific Ly6G antibody, as opposed to previous studies that have used nonspecific antibodies (Ly6C/G) against neutrophils and on different strains of A. baumannii, where the virulence of an isolate can influence the outcome (9). Our data with neutrophil-specific antibodies show specific and successful depletion (Figure 3, A and B, and see Supplemental Figure 9 for gating) that led to increased bacterial burden, irrespective of sex, in the lung and spleen (Figure $3 \mathrm{C}$ and Supplemental Figure 10). The magnitude of this increased bacterial burden compared with control mice was also equivalent between the sexes in the lung and spleen. Depletion of alveolar macrophages using clodronate-loaded liposomes was also successful (Figure 3, D and E, and see Supplemental Figure 9 for gating strategy), with no off-target cellular effects (Supplemental Figure 11). In contrast to neutrophils, depletion of alveolar macrophages, although increasing bacterial burdens in both sexes, had a disparately higher effect in bacterial counts in the airways of female mice (Figure 3F), with 2500-fold and 400-fold higher bacterial counts in the BALF and lung, respectively, compared with nondepleted controls, further highlighting the difference between sexes, whereas male mice had 9-, 8-, and 4-fold changes in the same sample types. Previous reports have shown that ROS production by neutrophils is a key factor in killing $A$. baumannii (31). In alveolar macrophage-depleted animals, males had significantly more ROS in the airway when compared with female mice (Figure 3G), which could contribute to their improved bacterial clearance compared with females. These results highlight an important role of alveolar macrophages in controlling $A$. baumannii pneumonia in female mice.

Female mice display metabolic changes in the absence of alveolar macrophages. To understand the differential sex effect of alveolar macrophage depletion, RNA-sequencing (RNA-Seq) of whole-lung homogenates of 
A
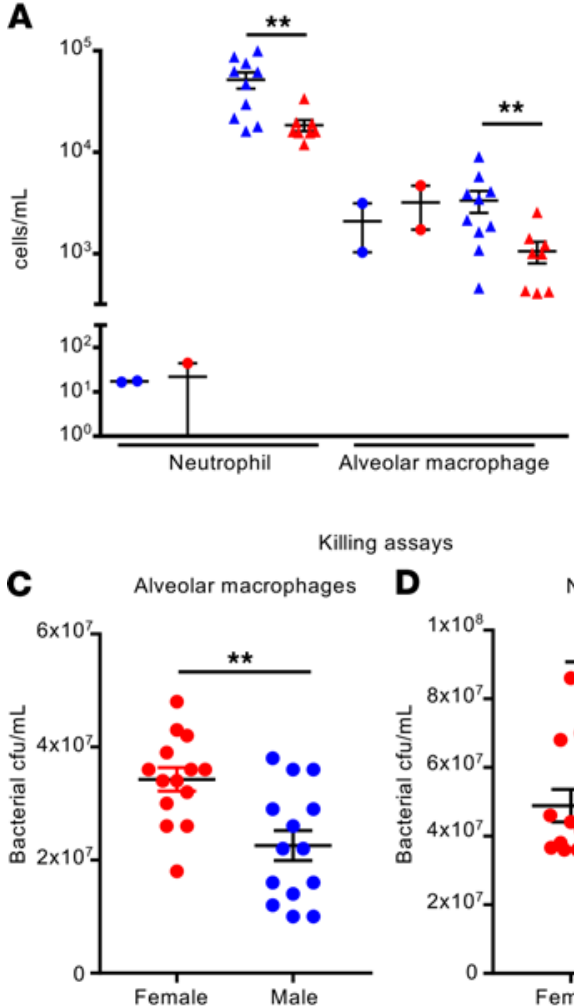

Killing assays

D

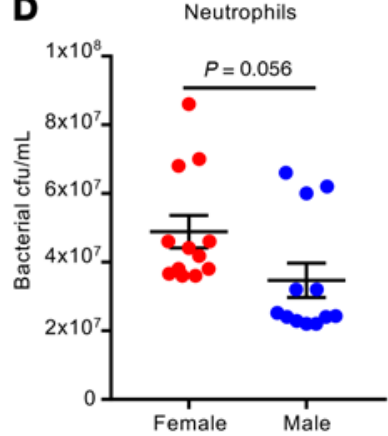

B $\log _{10} \mathrm{pg} / \mathrm{mL}$ High cytokines

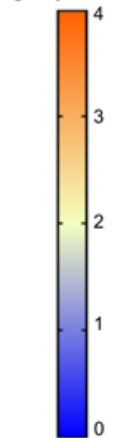

Low cytokines

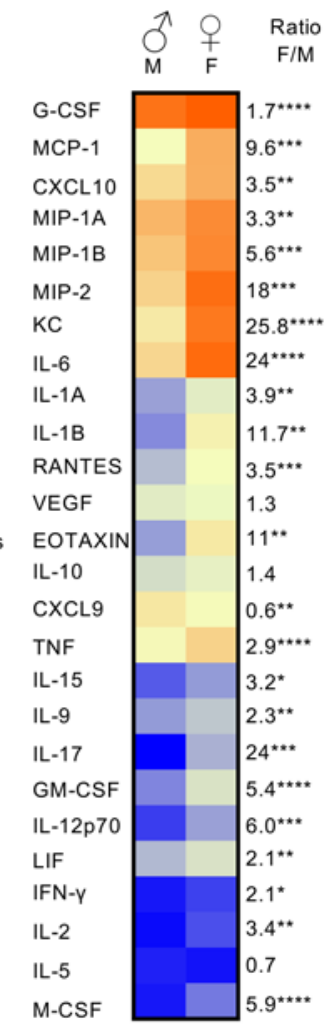
Ratio
- Male - PBS
- Female - PBS
$\triangle$ Male - A. baumannii
$\Delta$ Female - A. baumanni
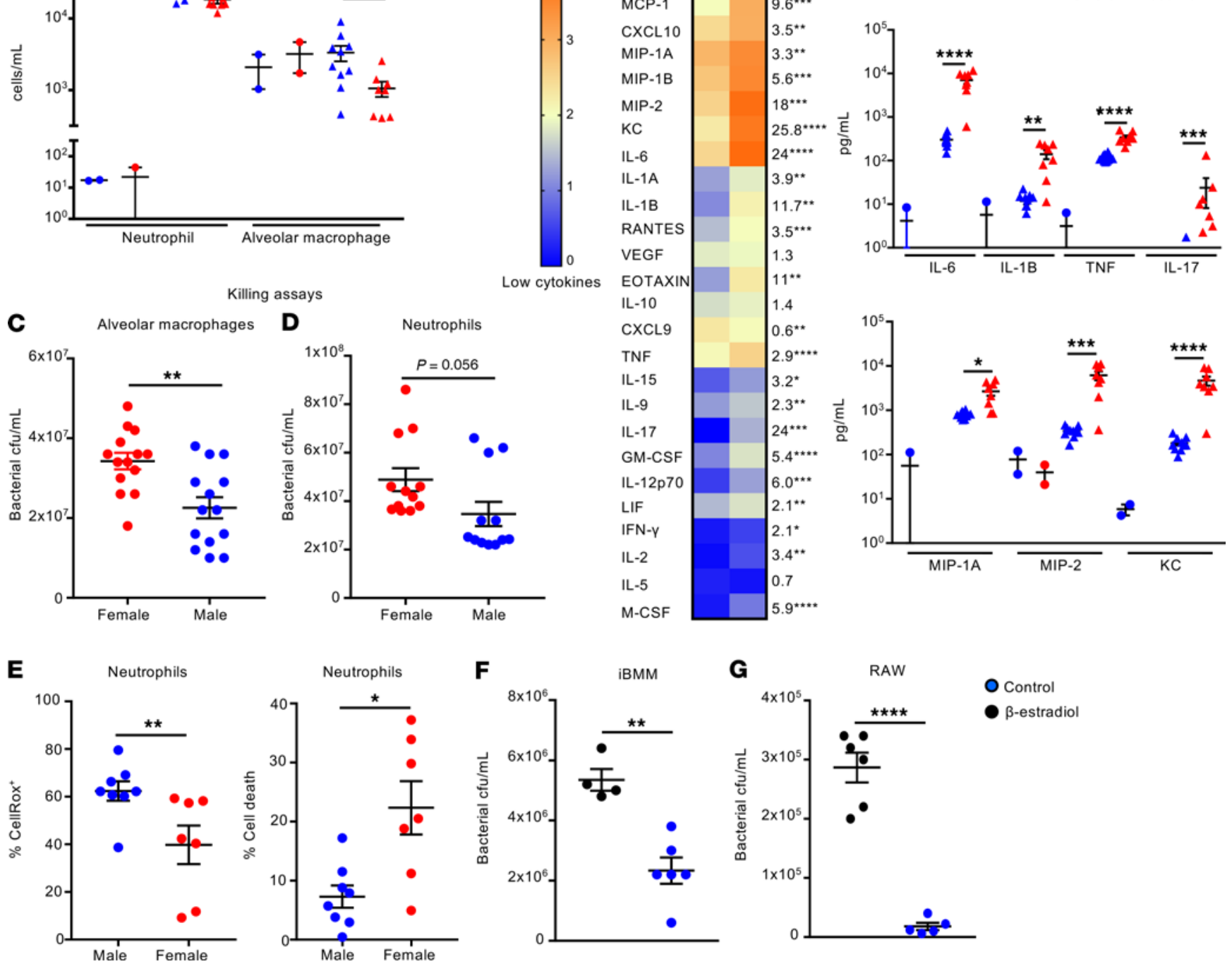

Figure 2. Female mice have exacerbated inflammation and reduced immune cell numbers in the airway in response to $\mathbf{A}$. baumannii.(A) Flow cytometry analysis of neutrophils and alveolar macrophages present in the airway of female and male weight-matched mice before and 24 hours after intranasal infection with $A$. baumannii. Infected mice: $n=8$ female, 10 male; $n=2$ uninfected. (B) Cytokine levels in BALF (infected mice: $n=8$ female, 10 male). Heatmap is $\log _{10}$ scale of cytokines in $\mathrm{pg} / \mathrm{mL}$, with female/male ratio and statistical significance. Select cytokines are shown individually. (C) Bacterial numbers were assessed 4 hours after infection of purified alveolar macrophages from both sexes of mice with $A$. baumannii $(n=14)$. (D) Bacterial numbers were assessed 1 hour after infection of bone marrow-derived neutrophils from female and male mice with $A$. baumannii $(n=12)$. (E) WT C57BL/6) mice were infected with $10^{7}$ CFU of $A$. baumannii for 24 hours, and neutrophils were treated with CellRox reagent to assess ROS production and cell death. $n=8$ males, and $n=7$ females. (F) Immortalized bone marrow macrophages (iBMMs) and (C) RAW macrophages were incubated with $\beta$-estradiol or vehicle overnight before incubating with $A$. baumannii and assessing killing 2 hours later. iBMM control $n=4$, $\beta$-estradiol $n=6$; RAW control $n=6$, $\beta$-estradiol $n=$ 5. Graphs show means with SEM. Data are from at least 2 independent experiments. ${ }^{*} P<0.05,{ }^{* *} P<0.01,{ }^{* *} P<0.001$, and ${ }^{* * * *} P<0.0001$. A nonparametric Mann-Whitney test $U$ was used to assess differences between groups.

clodronate-treated female and male mice following 4-hour infection was performed. We chose an early time point to minimize transcriptional changes due to substantial differences in bacterial burden between the sexes (Supplemental Figure 12A). Lung tissue was used to examine the entire effect on the lung in the absence of alveolar macrophages. Differences in bacterial burden between sexes were already apparent in BALF at 4 hours in clodronate-treated mice (Supplemental Figure 12A), which also correlated with a greater decrease in body temperature in female infected mice (Supplemental Figure 12B). Alveolar macrophages were significantly depleted from mice irrespective of sex (Supplemental Figure 12, C and D). RNA-Seq revealed significant changes between the sexes when alveolar macrophages were depleted (Figure 4A), with 
A

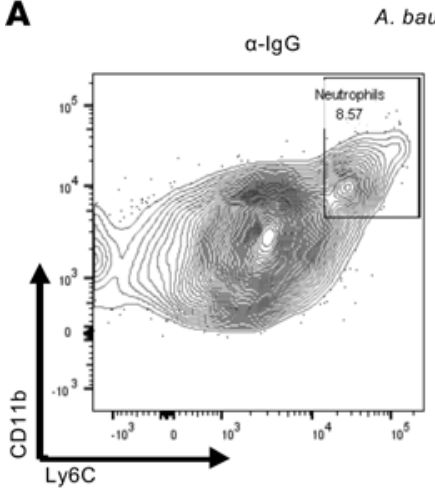

. baumannii

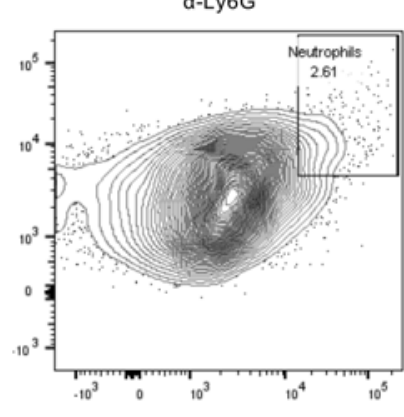

B

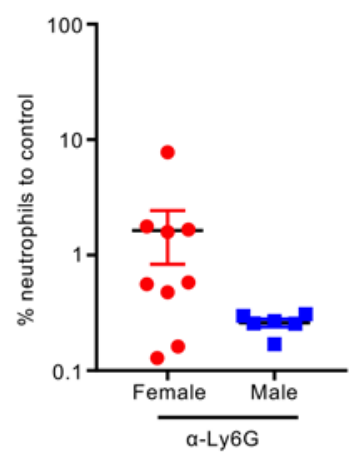

C

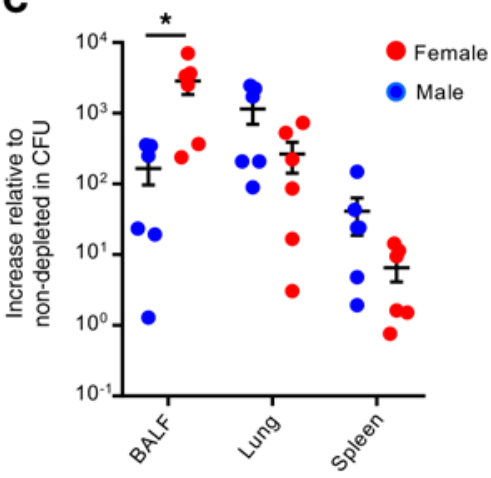

D

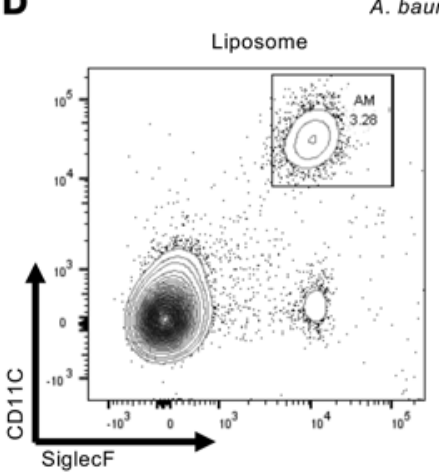

A. baumannii

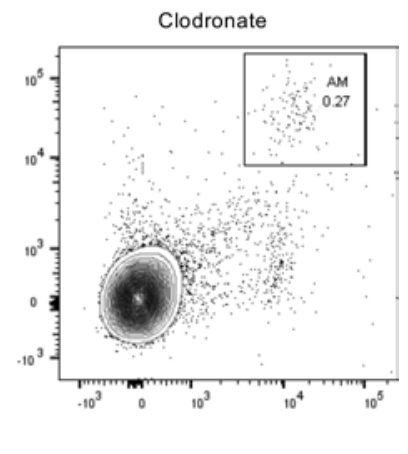

G ROS

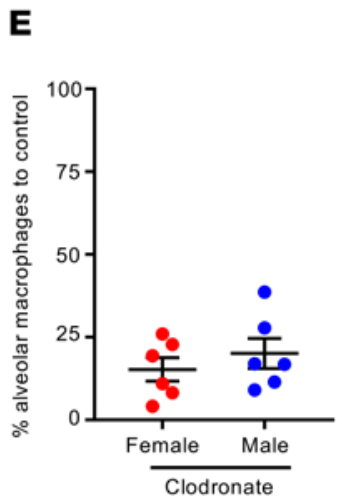

$\mathbf{F}$
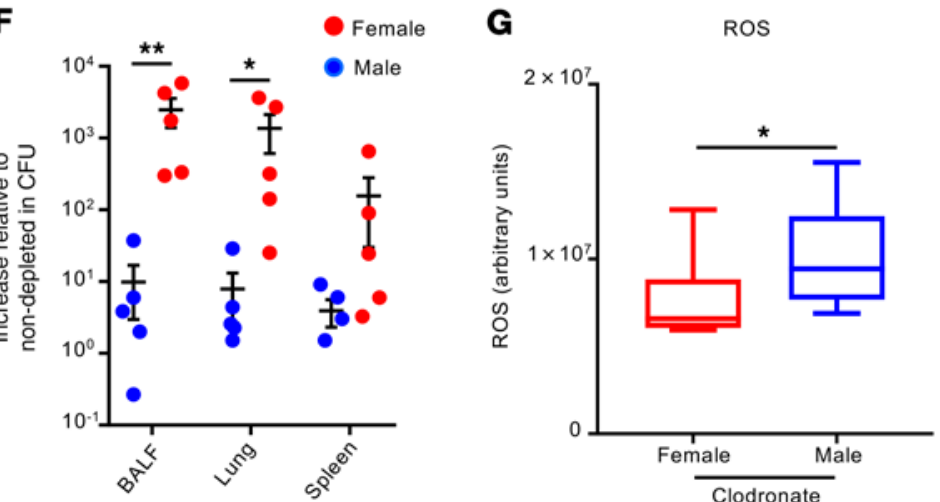

Figure 3. Depletion of alveolar macrophages results in a significantly higher bacterial burden in female than male infected mice. (A) Representative flow cytometry contour plot showing neutrophils in BALF of mice that were treated with either $\alpha$-IgG control or $\alpha$-Ly6G, then infected intranasally with $10^{7}$ CFU of $A$. baumannii. Neutrophils were identified as live CD45+SiglecF-MHCII-Ly6C+CD11b+. (B) Percentage of neutrophils in both female and male infected mice after neutrophil depletion relative to nondepleted levels. $n=6$. (C) Fold induction of bacterial numbers 24 hours after infection in female and male mice that were depleted of neutrophils compared with nondepleted controls. $n=6$. (D) Representative flow cytometry contour plot showing alveolar macrophages in BALF of mice treated with either liposome-only control or clodronate-loaded liposomes 24 hours prior to intranasal infection with $10^{7} \mathrm{CFU}$ of $A$. baumannii for 24 hours. Alveolar macrophages were identified as live $\mathrm{CD}^{2} 5^{+} \mathrm{Ly} \mathrm{C}^{-} \mathrm{CD} 11 \mathrm{C}^{+}$SiglecF $\mathrm{F}^{+}$. (E) Percentage of alveolar macrophages in both female and male infected mice after alveolar macrophage depletion relative to nondepleted levels. $n=6$. (F) Fold induction of bacterial numbers 24 hours after infection in female and male mice that were depleted of alveolar macrophages compared with nondepleted control. $n$ $=5$. (C) ROS levels in the airway of clodronate-treated female and male mice 24 hours after infection. $n=10$. Graphs show means with SEM. Each point represents a mouse. Data are from at least 2 independent experiments. ${ }^{*} P<0.05$, and ${ }^{* *} P<$ 0.01. A nonparametric Mann-Whitney $U$ test was used to assess differences between groups.

over 2500 (fold change $>1.5 ; P<0.05$ ) transcript changes (Figure 4B). We observed significantly fewer gene changes in the absence of alveolar macrophage depletion at this time point (Supplemental Figure 13). We observed an increase of 952 transcripts in female mice and 1647 transcript decreases (Figure 4B), suggesting that alveolar macrophages in female mice contribute substantially to the transcriptional landscape. 
Gene ontology analysis showed major reductions in the respiratory chain and ribosomal genes in female infected mice, indicating that alveolar macrophages from female mice contribute significantly to energetic requirements (Figure 4C). Alterations in mitochondrial complex function can propel the cell to altered ROS generation. Total ROS levels were not altered at this time point (Supplemental Figure 12F); however, transcription of several genes involved in the antioxidant response system, such as Sod1 and Sod3 (data not shown), was upregulated in male mice, suggesting that male mice can have increased protection against ROS. LDH quantification revealed a trend toward increased cytotoxicity and cell death in female mice, although this was not significant (Supplemental Figure 12G, $P=0.0976$ ). Given that mitochondria are the primary energy producer, ATP levels were measured in BALF. Consistent with the transcriptional data, female mice displayed a significant decrease of $92 \%$ in total ATP levels when compared with male mice (Figure 4D, $P<0.05$ ). Lactate levels, which are a common readout for glycolysis (32), were increased by $42 \%$ in female mice (Figure $4 \mathrm{E}, P<0.05$ ) 4 hours following infection, indicating that due to reductions in mitochondrial oxidative phosphorylation, female mice need to switch to a glycolytic state in order to generate ATP. We confirmed the importance of this observation by inhibiting cellular respiration in mice with rotenone, an inhibitor of mitochondrial electron transport. Treatment of male mice with this compound increased bacterial burdens by 16 -fold $(P<0.05$; Figure $4 \mathrm{~F})$ in BALF and 5.3 -fold in lung tissue $(P$ $<0.01$; Figure $4 \mathrm{~F})$. These data suggest that in the absence of alveolar macrophages, female mice are unable to respond in a metabolically beneficial manner to A. baumannii infection.

\section{Discussion}

In this study we identified a sex-based innate immune response to A. baumannii respiratory tract infection. We showed that female mice are significantly more susceptible to infection, irrespective of $A$. baumannii virulence, which was also mimicked in male mice treated with $17 \beta$-estradiol. We also demonstrated that the differential susceptibility to $A$. baumannii-induced pneumonia between sexes is based on altered immune cell populations in the airway, specifically neutrophils, and depletion of alveolar macrophages leads to a metabolic impairment of female mice following infection, also hindering their capacity to combat infection.

Previous work has pointed to an important role for alveolar macrophages in the early control of $A$. baumannii replication in the airway, possibly by contributing to the recruitment of neutrophils via production of inflammatory cytokines and chemokines (27). In this report we showed that alveolar macrophage depletion in female mice resulted in a decrease of the eukaryotic initiation factor 2 initiation machinery, possibly resulting in decreased protein translation as well as severe alterations in mitochondria, which led to metabolic dysfunctions. A recent report raises the hypothesis of sex-dependent actin cytoskeleton regulation in alveolar macrophages (33), which has the potential to affect several processes, such as phagocytosis. Here we reveal another layer on innate immune sex-based differences by demonstrating that alveolar macrophages in female mice infected with $A$. baumannii are important to maintain metabolic homeostasis and facilitate an effective response to infection.

Defining the role of neutrophils in A. baumannii respiratory infection has up until now relied on nonspecific antibodies $(26,30)$. In our model, neutrophil depletion eliminates the sex difference associated with higher bacterial burdens, suggesting that the decreased neutrophil numbers observed in the airway of the female mice are a crucial factor in mediating this phenotype. Moreover, clodronate-treated female mice already had $35 \%$ fewer neutrophils in the airway than male mice 4 hours after infection (Supplemental Figure 12E, $P=$ 0.0952), thus suggesting that alveolar macrophages can regulate neutrophil numbers in the airway in response to $A$. baumannii infection. Neutrophil-derived ROS has been described as a key factor in the killing of $A$. baumannii in respiratory tract infection (31). Despite conflicting results in the literature on the role of estradiol in neutrophils and ROS production, recent work shows that estradiol could lead to a reduction of superoxide anion production by neutrophils (34). We observed that ROS levels were increased in the airway of male mice 24 hours after infection (Figure 3G) and their levels were higher in neutrophils from male mice (Figure 2G), thus contributing to the improved bacterial clearance. Also, while female mice had $64 \%$ fewer neutrophils than male mice, they had a significant increase in neutrophil chemokine (Figure 2, A and B) levels in the airway. When neutrophil transmigration to inflamed tissues is blocked, IL-17 production is induced, which induces G-CSF production, causing increased granulopoiesis and numbers of circulating neutrophils $(35,36)$. We observed a significant increase in the transcription of Il17a (2.5-fold) and Csf3 (1.95-fold) in female mice (Supplemental Figure 12H). All together our results suggest that neutrophil migration and ROS production and survival in the airway might be impaired in female mice. 
A

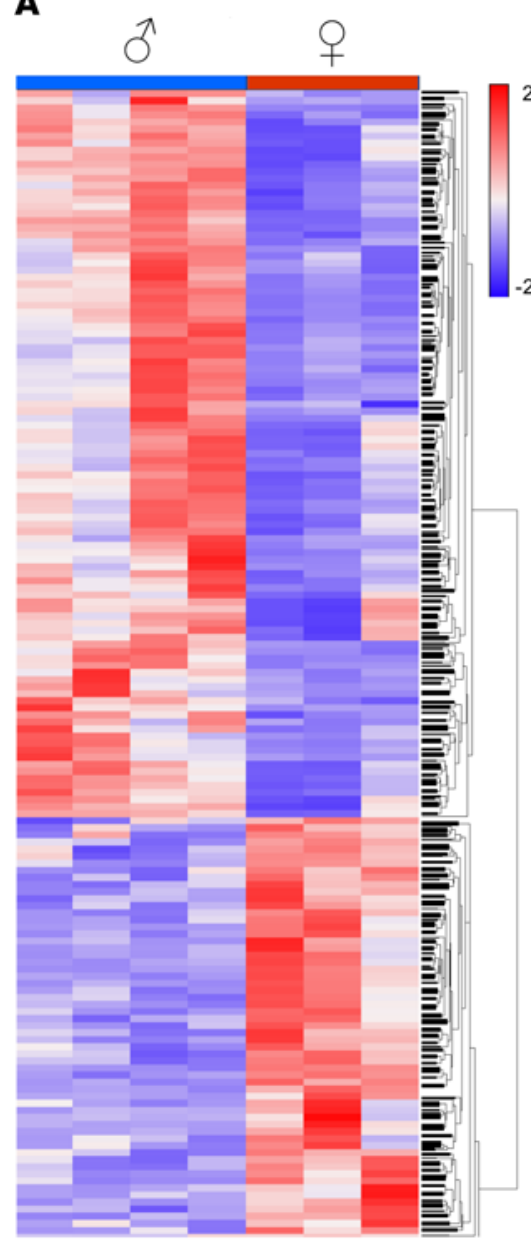

B

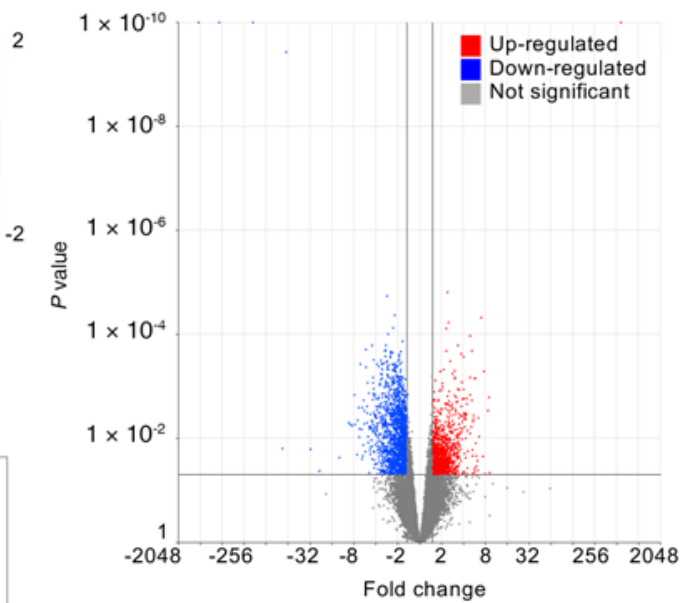

C
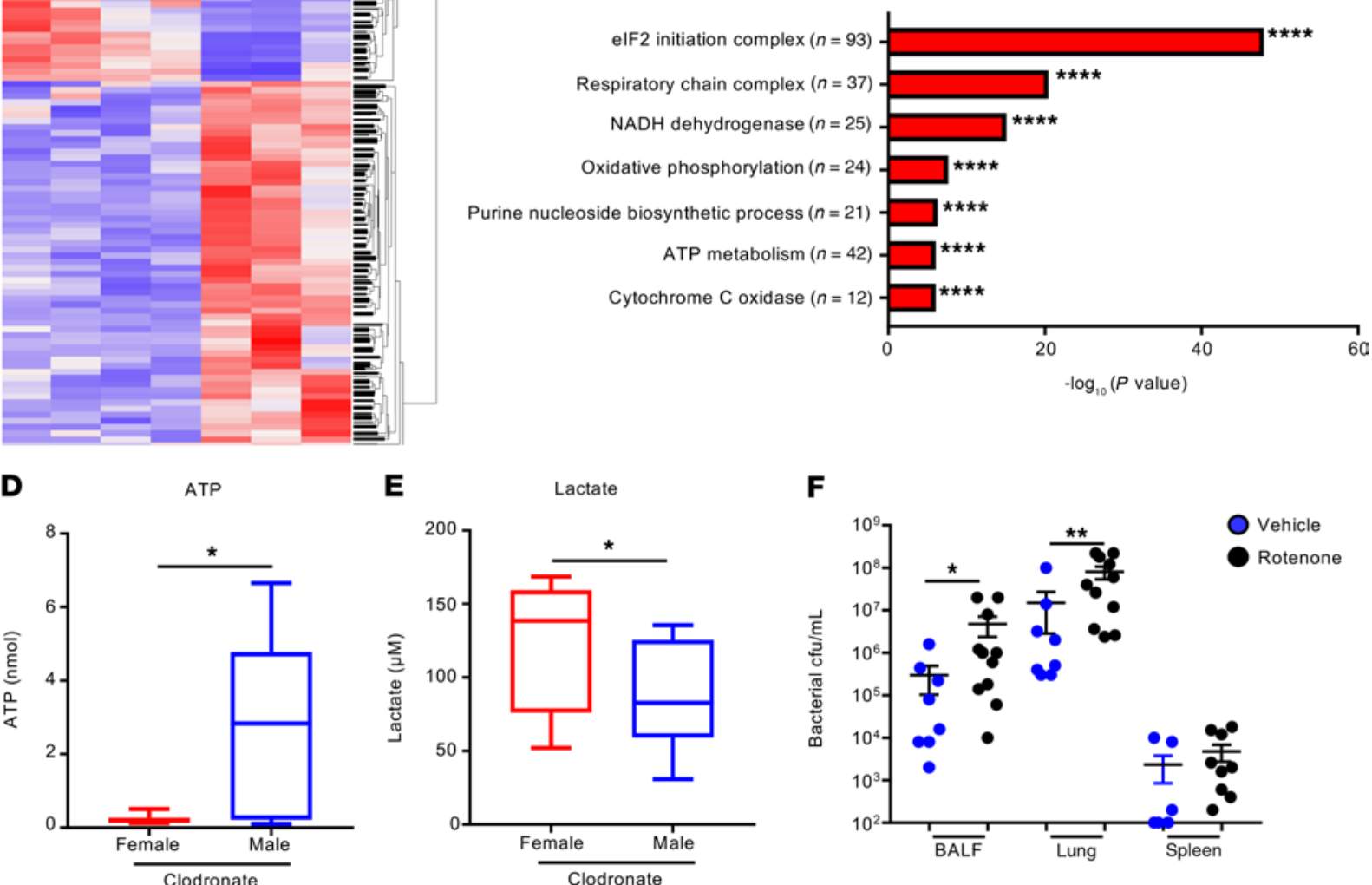

Figure 4. Alveolar macrophage depletion results in mitochondrial defects and energetic imbalance in female infected mice. (A) Heatmap of RNA-Seq analysis of the comparison between female and male mice, depleted of alveolar macrophages, following a 4-hour infection. Each column represents an individual mouse. (B) Volcano plot of differentially expressed genes. (C) Gene ontology analysis of differentially expressed genes that are downregulated in female mice compared with males. (D and E) ATP and lactate levels, respectively, in BALF following a 4-hour infection in female and male mice depleted of alveolar macrophages. $n=12$. Box-and-whisker plots show mean with upper and lower quartiles, with whiskers at minimum and maximum values. (F) Bacterial counts from BALF, lung, and spleen of $A$. baumannii-infected mice that had been treated with rotenone or vehicle control. Control $n=8$; rotenone $n=11$. Graphs show means with SEM. Data are from 2 independent experiments. ${ }^{*} P<0.05$, ${ }^{* *} P<0.01$, and ${ }^{* * * *} P<$ 0.0001. A nonparametric Mann-Whitney $U$ test was used to assess differences between groups.

The majority of studies do not report the use of both sexes of mice (37), including those investigating the innate immune response to $A$. baumannii pneumonia. Two previous reports made side observations of increased bacterial burden in female mice, but the underlying causes were not pursued $(38,39)$. In one study, female mice infected similarly as in our study displayed increased disease when compared with male 
mice, and this was true for both C57BL/6 and BALB/c mice (38). C57BL/6 mice were more susceptible to $A$. baumannii than BALB/c mice, which has been attributed in other infections to increased neutrophil influx and increased phagocytic capacity and oxidative burst of alveolar macrophages in BALB/c mice (40). Another open question and area for future investigation is how the female immune system directly influences the immune response to A. baumannii. Studies in humans are limited. One study showed a significant predominance of infection with the male population; however, this did not go into detail regarding severity of infection and patient outcome (41). Alternatively, this could be evidence of a fundamental difference that does not agree between mice and humans.

It has been seen in the human female population a bias exists toward several infectious diseases. These infections include Legionella pneumophila, HIV, malaria, and influenza (12). In mice, greater mortality is observed in female mice for influenza infection, with estrogen leading to a higher inflammatory response (42), akin to what we observed in female mice infected with $A$. baumannii. In humans, evidence exists that females fare worse for several lung-inflammatory conditions, including cystic fibrosis, chronic obstructive pulmonary disease, asthma, and non-CF-related bronchiectasis $(43,44)$. Potential mechanisms behind these biases have included genetic predisposition, comorbidities, and sex hormones. In female asthmatics, for example, lung function is at its lowest during the luteal phase of the menstrual cycle, when there is high estrogen and progesterone (45). Direct evidence as to how the hormones influence these conditions is not fully known. While our own respiratory studies with Staphylococcus aureus, Klebsiella pneumoniae, or Streptococcus pneumoniae (46-49) have not indicated a significant susceptibility for any particular sex in mouse models, it has been shown that female mice are more susceptible to respiratory Pseudomonas aeruginosa infection (15). Consistent with our observations, female mice intranasally infected with $P$. aeruginosa have increased rates of mortality and levels of inflammatory cytokines, in addition to increased neutrophil necrosis (15). We observed that depletion of neutrophils led to increased bacterial burdens in our study irrespective of sex, so the observed increase in neutrophil necrosis in females both in our study and Abid et al. (15) is a likely reason behind the sex bias. Why this is not observed in other bacterial respiratory models in mice could be due to differences in animal models and humans. Patients with neutropenia are at much greater risk of $S$. aureus pneumonia (50), yet depletion of neutrophils in mice does not change bacterial burden (51), unlike what we observed with $A$. baumannii.

Estrogen has also been shown to influence nutritional immunity. In the host, iron homeostasis is controlled by the hepcidin/ferroportin axis, with hepcidin acting as the master regulator. It has been documented that there is an estrogen response element in the promoter region of hepcidin $(52,53)$, such that exogenous addition of estrogen leads to its repression (54). In mice lacking estrogen there are less hemoglobin and reduced iron levels in the serum. Because iron is an essential nutrient, reduced iron could be a mechanism behind sex bias in bacterial infections. Also, in male mice treated with estrogen, a significant reduction in lactoferrin was observed in the context of pulmonary $P$. aeruginosa infection (55). Lactoferrin is an important iron-chelating antimicrobial compound because its absence increases the available free iron available to pathogens. Lactoferrin has also been shown to be able to inhibit $P$. aeruginosa biofilm formation (56), an important aspect of its pathogenic life cycle. Although we did not specifically test for lactoferrin, as with most organisms, iron is an important aspect of $A$. baumannii pathogenesis (57) and is a potential treatment strategy (58) and could also be a reason behind our observed sex phenotype.

Our findings reflect a fundamental difference in the innate immune response to $\mathrm{A}$. baumannii infection in the airway between female and male mice. By gaining a better understanding of the host response, we may identify elements of the immune system that could be targets for immunomodulatory therapy. Therefore, given these results, studies moving forward should address differences between the sexes. By identifying sex-related immune differences, preclinical models can be designed appropriately to control for these differences and better develop therapeutics efficacious to both sexes. These sex studies have potential implications between sexes and males receiving hormone therapy in the general population.

\section{Methods}

Bacterial culture. A. baumannii strains 5075 and 19606 were grown in LB broth at $37^{\circ} \mathrm{C}$ to exponential phase (optical density of 1.0 at $600 \mathrm{~nm}$ ). Growth curves were carried out in $\mathrm{LB}$ and $\mathrm{M} 9$ media with $2 \mathrm{mM} \mathrm{MgSO}_{4}, 0.1$ $\mathrm{mM} \mathrm{CaCl}_{2}, 0.1 \%$ casamino acids, and $0.5 \%$ glucose with $20 \mu \mathrm{g} / \mathrm{mL}$ of $\beta$-estradiol or ethanol vehicle control.

Animal studies. Female and male C57BL/6J WT mice, 6 to 8 weeks old, were intranasally infected while under anesthesia with $1 \times 10^{7} \mathrm{CFU}$ of $A$. baumannii for 4 hours or 24 hours. For mortality studies C57BL/6 WT mice were intranasally infected with $10^{8} \mathrm{CFU}$ of $A$. baumannii and mortality was assessed for 7 days. 
Weight-matched mice were purchased from The Jackson Laboratory. Male mice were treated with either $17 \beta$-estradiol (10 $\mu \mathrm{g} /$ mouse; MilliporeSigma) or vehicle control (sesame oil; MilliporeSigma) intraperitoneally, daily for 5 days before infection with $A$. baumannii. Respiration inhibition was conducted using $8 \mathrm{mg} / \mathrm{kg}$ of rotenone 4 hours prior to infection (59). Lungs were fixed in $4 \%$ paraformaldehyde overnight to prepare paraffin blocks for H\&E-stained slides. Images were taken using a Keyence BZ-X710 microscope. BALF was obtained from 3 successive washes with $1 \mathrm{~mL}$ of PBS. BALF was used to enumerate bacterial counts, quantify cytokine levels (mouse 32-plex; Eve Technologies), stain cells for flow cytometry, and assess protein content using Bradford reagent. Lung and spleen tissue was homogenized in $400 \mu \mathrm{L}$ of PBS and used to enumerate bacterial counts. Temperature was quantified using an external infrared thermometer.

Flow cytometry. Cells in BALF were stained with fluorescently labeled antibodies: CD45-Alexa Fluor 700 (30-F11), CD11b-PE-Cy7 (M1/70), Ly6G-PerCP-Cy5.5 (1A8), CD11c-BV605 (N418), CD45-Alexa Fluor 700 (30-F11), BV510-CD103 (2E7), SiglecF-Alexa Fluor 647 (E50-2440; BD Biosciences), MHCII-APC-Cy7 (M5/114.15.2), Ly6C-PE-Texas red (AL-2; BD Biosciences), and DAPI to assess cell viability. Antibodies were from BioLegend unless otherwise stated. Cells in the airway were classified as follows: neutrophils - live

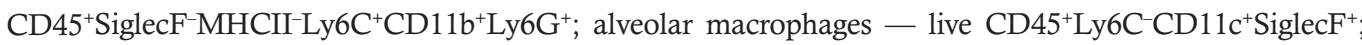
eosinophils - live $\mathrm{CD}^{2} 5^{+} \mathrm{Ly} 6 \mathrm{C}^{-} \mathrm{CD} 11 \mathrm{c}^{-}$SiglecF $\mathrm{CD}^{+} \mathrm{Cb}^{+}$; interstitial macrophages - live $\mathrm{CD}^{2} 5^{+} \mathrm{Ly} 6 \mathrm{C}^{-} \mathrm{Si}$ -

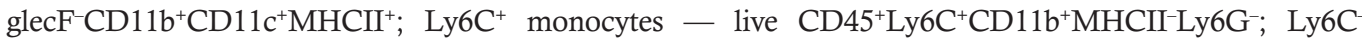
monocytes - live $\mathrm{CD}^{2} 5^{+} \mathrm{Ly} \mathrm{C}^{-}$SiglecF $\mathrm{CD}^{-} \mathrm{CD} 11 \mathrm{~b}^{+} \mathrm{CD} 11 \mathrm{c}^{+} \mathrm{MHCII}^{-}$; plasmacytoid dendritic cells (DCs) - live

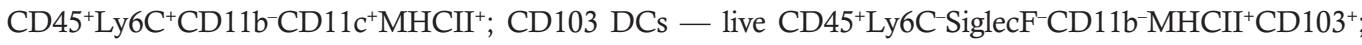
and $\mathrm{CD} 11 \mathrm{~b}$ DCs - live $\mathrm{CD} 45^{+} \mathrm{Ly} 6 \mathrm{C}^{+} \mathrm{CD} 11 \mathrm{~b}^{+} \mathrm{MHCII}{ }^{+} \mathrm{CD} 11 \mathrm{c}^{+}$. ROS production and cell death in cells were measured using CellRox (Life Technologies).

Cell depletion. Alveolar macrophages were depleted by inoculating intranasally anesthetized mice with $50 \mu \mathrm{L}$ of either liposome containing clodronate $(5 \mathrm{mg} / \mathrm{mL})$ or liposome only (Encapsula NanoSciences) 24 hours before infection with $A$. baumannii. Efficiency of depletion was assessed by flow cytometry. To deplete neutrophils, mice were injected intraperitoneally either with $250 \mu \mathrm{g}$ of anti-mouse Ly6G (clone 1A8; Bio X Cell) or with IgG2a, $\kappa$ isotype control (clone 2A3; Bio X Cell), 24 hours before infection. Efficiency of depletion was assessed by flow cytometry.

Cell culture. Neutrophils were isolated from the bone marrow of the femurs and tibias of naive female and male mice by density gradient centrifugation as previously described (48). Bone marrowderived neutrophils were used for experiments on the day of purification. To evaluate bacterial killing capacity, neutrophils were incubated with A. baumannii (multiplicity of infection [MOI] of 10) for 60 minutes with slow rotation at $37^{\circ} \mathrm{C}$, after which the reaction was stopped at $4^{\circ} \mathrm{C}$ and cells were lysed with $1 \%$ saponin. Bacterial numbers were determined by serial dilutions. Alveolar macrophages were purified from the airway of naive female and male mice by bronchoalveolar lavage. To evaluate bacterial killing capacity, alveolar macrophages were incubated with $A$. baumannii (MOI of 10) for 4 hours, after which intracellular bacterial numbers were determined by serial dilutions. iBMMs (EI Resources) and RAW cells (provided by George Yap, Center for Immunity and Inflammation, Rutgers New Jersey Medical School) were cultured in RPMI 1640 medium with penicillin, streptomycin, and 10\% heat-inactivated fetal bovine serum. Cells were seeded and treated with $100 \mathrm{nM}$ of $\beta$-estradiol 2 hours prior to incubation with $A$. baumannii (MOI 10), at which point bacterial numbers were quantified.

ATP and lactate assays. ATP and lactate levels were measured in BALF samples from clodronate-depleted female and male mice following a 4-hour infection with $A$. baumannii. ATP levels were determined using the ATP Colorimetric/Fluorometric Assay Kit (BioVision, Inc.) following the manufacturer's instructions. Lactate levels were determined using the fluorimetric EnzyFluo L-Lactate Assay Kit (BioAssay Systems) following the manufacturer's instructions. To avoid degradation of lactate by the presence of endogenous lactate dehydrogenase $(\mathrm{LDH})$, samples were filtered through a $10-\mathrm{kDa}$ MW spin filter to remove all proteins.

LDH assay. In BALF samples from clodronate-depleted female and male mice following a 4-hour infection with $A$. baumannii, LDH was determined using the CytoSelect LDH Cytotoxicity Assay Kit (Cell Biolabs, Inc.) and following the manufacturer's instructions.

ROS measurement. ROS levels were measured in BALF samples from clodronate-depleted female and male mice following a 4-hour and 24-hour infection with $A$. baumannii. Carboxy-H2DCFDA (Molecular Probes, Thermo Fisher Scientific) was added to $50 \mu \mathrm{L}$ of BALF to a final concentration of $10 \mu \mathrm{M}$, and carboxydichlorofluorescein fluorescence was read at excitation $495 \mathrm{~nm} /$ emission $520 \mathrm{~nm}$ after 1 hour. 
RNA-Seq. RNA was extracted from whole lungs using the Direct-zol RNA MiniPrep Kit (Zymo Research), following the manufacturer's instructions. RNA-Seq was performed by Novogene. mRNA was purified from $1 \mu \mathrm{g}$ of total RNA using poly-T oligo-attached magnetic beads and then fragmented using fragmentation buffer. The first strand of cDNA was synthesized using a random hexamer and M-MuLV reverse transcriptase with the second strand of cDNA synthesized using DNA polymerase I and RNase H. cDNA was purified using AMPure XP beads (Beckman Coulter), converted to blunt ends, adenylated, and New England Biolabs adaptors ligated. Sequencing was performed on a NovoSeq 6000 (Illumina) with 20 million paired end $150 \mathrm{bp}$ reads.

Sequencing data were analyzed using Partek. Reads were trimmed with Phred scores less than 30, 20 bp minimum read length, and a maximum $\mathrm{N}$ of $10 \%$. Adaptor sequences were removed. Trimmed reads were aligned using STAR and unaligned reads further aligned using TopHat2. Combined alignments were quantified to the annotation model (Partek), and low read counts $(\mathrm{N}<10)$ were filtered. Filtered counts were normalized by adding 1 and using trimmed mean of M-values normalization. Differential analysis was conducted using ANOVA and Limma-voom. Genes with $P<0.05$ and fold changes $>1.5$ were used for gene set enrichment using Partek and Panther. Hierarchical clustering was based on the union of differentially expressed genes across all comparisons using an average linkage cluster metric and Euclidean point distance metric. RNA-Seq data were deposited into the National Center for Biotechnology Information's Gene Expression Omnibus database (GSE134805).

Microbiome analysis. Mouse fecal pellet samples were thawed to room temperature from the $-80^{\circ} \mathrm{C}$ freezer and DNA extracted using the QIAamp PowerFecal DNA Kit according to the manufacturer's instructions (QIAGEN). A column with no fecal pellet was included as a DNA-free control. Extracted DNA was quantified using a Qubit 3.0 fluorometer and the Qubit dsDNA HS Assay Kit (Thermo Fisher Scientific). Isolated mouse fecal pellet DNA samples were used to prepare libraries according to the Illumina protocol for 16S Metagenomics Sequencing Library Preparation (15044223 B). The V3 and V4 regions of the $16 \mathrm{~S}$ rRNA were amplified using Illumina 16S amplicon primers (forward primer TCGTCGGCAGCGTCAGATGTGTATAAGAGACAGCCTACGGGNGGCWGCAG and reverse primer GTCTCGTGGGCTCGGAGATGTGTATAAGAGACAGGACTACHVGGGTATCTAATCC) using the 2X KAPA HiFi HotStart ReadyMix kit (Kapa Biosystems) and the 16S rRNA amplicons purified using AMPure XP beads (Beckman Coulter). Index PCR was performed using the Nextera DNA CD Indexes kit (Illumina) with 2X KAPA HiFi HotStart ReadyMix, and indexed PCR products were purified as before using AMPure beads. The indexed libraries were quantified using the Qubit 3.0 and Qubit dsDNA HS Assay Kit and diluted in $10 \mathrm{mM}$ Tris- $\mathrm{HCl}$ to a final concentration of approximately $0.3 \mathrm{ng} /$ $\mu \mathrm{L}(1 \mathrm{nM})$. A pool containing $5 \mu \mathrm{L}$ of each library was generated, and $5 \mu \mathrm{L}$ of the pool was diluted in $95 \mu \mathrm{L}$ of $10 \mathrm{mM}$ Tris- $\mathrm{HCl}$ to make a final pooled library concentration of $50 \mathrm{pM}$. Before sequencing on the iSeq, a 10\% spike in $50 \mathrm{pM}$ PhiX control v3 (Illumina) was added to the pooled library; $20 \mu \mathrm{L}$ of the pooled library with PhiX spike was loaded onto the iSeq. Sequencing data were uploaded to the BaseSpace Sequence Hub, and reads were demultiplexed using a FASTQ generation script. The run yielded $2.05 \mathrm{Gbp}$ with an average quality score 30 of $91.8 \%$. QIIME2 was used to assign taxonomic units to $16 \mathrm{~S}$ rRNA sequences using Divisive Amplicon Denoising Algorithm (DADA2) to filter and infer bacterial taxa to amplicons (60). Taxonomic units were assigned to DADA2 feature IDs using the Silva taxonomy classifier (61). Principal component analysis and extended bar plots representing the taxonomic diversity of samples were performed using statistical analysis of taxonomic and functional profiles (62). Processed sequence reads were submitted to Sequence Read Archive (BioProject PRJNA560086).

Statistics. Animal data were assessed using a nonparametric Mann-Whitney $U$ test. In vitro experiments were assessed using an unpaired 2-tailed Student's $t$ test. A $P$ value less than 0.05 was considered significant. Statistical analyses were performed with Prism software (GraphPad).

Study approval. Animal work in this study was carried out in strict accordance with the recommendations in the Guide for the Care and Use of Laboratory Animals of the NIH (National Academies Press, 2011), the Animal Welfare Act, and US federal law. Protocols were approved by the Institutional Animal Care and Use Committee of Rutgers New Jersey Medical School of Newark, New Jersey, USA.

\section{Author contributions}

SP and DP designed and conducted the experiments, analyzed the data, and wrote the manuscript. AP conducted experiments. JS and DSS extracted DNA from fecal matter and conducted the 16S rRNA analysis of the microbiome. DP provided funding. 


\section{Acknowledgments}

The authors thank Tessa Bergsbaken for helpful discussions and Joan Durbin for expert pathology analysis. This work was supported by The New School to DSS and Rutgers University and NIH R01HL134870 to DP.

Address correspondence to: Dane Parker, Center for Immunity and Inflammation, Rutgers New Jersey Medical School, 205 South Orange Ave., G-1208, Newark, New Jersey 07103, USA. Email: dane.parker@rutgers.edu. SP's present address is: Department of Medicine, Weill Cornell Medicine, New York, New York, USA.

1. Antunes LC, Visca P, Towner KJ. Acinetobacter baumannii: evolution of a global pathogen. Pathog Dis. 2014;71(3):292-301

2. Dijkshoorn L, Nemec A, Seifert H. An increasing threat in hospitals: multidrug-resistant Acinetobacter baumannii. Nat Rev Microbiol. 2007;5(12):939-951.

3. Gallagher LA, et al. resources for genetic and genomic analysis of emerging pathogen Acinetobacter baumannii. $J$ Bacteriol. 2015;197(12):2027-2035.

4. Göttig S, Gruber TM, Higgins PG, Wachsmuth M, Seifert H, Kempf VA. Detection of pan drug-resistant Acinetobacter baumannii in Germany. J Antimicrob Chemother. 2014;69(9):2578-2579.

5. Li YJ, et al. Pneumonia caused by extensive drug-resistant Acinetobacter baumannii among hospitalized patients: genetic relationships, risk factors and mortality. BMC Infect Dis. 2017;17(1):371.

6. Özgür ES, Horasan ES, Karaca K, Ersöz G, Naycı Atış S, Kaya A. Ventilator-associated pneumonia due to extensive drug-resistant Acinetobacter baumannii: risk factors, clinical features, and outcomes. Am J Infect Control. 2014;42(2):206-208.

7. Wu HG, Liu WS, Zhu M, Li XX. Research and analysis of 74 bloodstream infection cases of Acinetobacter baumannii and drug resistance. Eur Rev Med Pharmacol Sci. 2018;22(6):1782-1786.

8. García-Patiño MG, García-Contreras R, Licona-Limón P. The immune response against Acinetobacter baumannii, an emerging pathogen in nosocomial infections. Front Immunol. 2017;8:441.

9. Pires S, Parker D. Innate immune responses to Acinetobacter baumannii in the airway. JInterferon Cytokine Res. 2019;39(8):441-449.

10. Klein SL, Jedlicka A, Pekosz A. The Xs and Y of immune responses to viral vaccines. Lancet Infect Dis. 2010;10(5):338-349.

11. Dunn SE, et al. Peroxisome proliferator-activated receptor (PPAR)alpha expression in T cells mediates gender differences in development of T cell-mediated autoimmunity. J Exp Med. 2007;204(2):321-330.

12. Klein SL, Flanagan KL. Sex differences in immune responses. Nat Rev Immunol. 2016;16(10):626-638.

13. Voskuhl R. Sex differences in autoimmune diseases. Biol Sex Differ. 2011;2(1):1.

14. Scotland RS, Stables MJ, Madalli S, Watson P, Gilroy DW. Sex differences in resident immune cell phenotype underlie more efficient acute inflammatory responses in female mice. Blood. 2011;118(22):5918-5927.

15. Abid S, et al. 17ß-estradiol dysregulates innate immune responses to Pseudomonas aeruginosa respiratory infection and is modulated by estrogen receptor antagonism. Infect Immun. 2017;85(10):e00422-17.

16. Vermillion MS, Ursin RL, Attreed SE, Klein SL. Estriol reduces pulmonary immune cell recruitment and inflammation to protect female mice from severe influenza. Endocrinology. 2018;159(9):3306-3320.

17. Dikshit N, et al. NLRP3 inflammasome pathway has a critical role in the host immunity against clinically relevant Acinetobacter baumannii pulmonary infection. Mucosal Immunol. 2018;11(1):257-272.

18. de Breij A, Eveillard M, Dijkshoorn L, van den Broek PJ, Nibbering PH, Joly-Guillou ML. Differences in Acinetobacter baumannii strains and host innate immune response determine morbidity and mortality in experimental pneumonia. PLoS One. 2012;7(2):e30673.

19. Khan N, et al. Intestinal dysbiosis compromises alveolar macrophage immunity to Mycobacterium tuberculosis. Mucosal Immunol. 2019;12(3):772-783.

20. Schuijt TJ, et al. The gut microbiota plays a protective role in the host defence against pneumococcal pneumonia. Gut. 2016;65(4):575-583.

21. Gauguet S, et al. Intestinal microbiota of mice influences resistance to Staphylococcus aureus pneumonia. Infect Immun. 2015;83(10):4003-4014.

22. Liggins M, Ramirez N, Magnuson N, Abel-Santos E. Progesterone analogs influence germination of Clostridium sordellii and Clostridium difficile spores in vitro. J Bacteriol. 2011;193(11):2776-2783.

23. Chotirmall SH, et al. Effect of estrogen on pseudomonas mucoidy and exacerbations in cystic fibrosis. $N$ Engl J Med. 2012;366(21):1978-1986.

24. Edwards JL. Neisseria gonorrhoeae survival during primary human cervical epithelial cell infection requires nitric oxide and is augmented by progesterone. Infect Immun. 2010;78(3):1202-1213

25. Kadel S, Kovats S. Sex hormones regulate innate immune cells and promote sex differences in respiratory virus infection. Front Immunol. 2018;9:1653.

26. van Faassen H, KuoLee R, Harris G, Zhao X, Conlan JW, Chen W. Neutrophils play an important role in host resistance to respiratory infection with Acinetobacter baumannii in mice. Infect Immun. 2007;75(12):5597-5608.

27. Qiu H, KuoLee R, Harris G, Van Rooijen N, Patel GB, Chen W. Role of macrophages in early host resistance to respiratory Acinetobacter baumannii infection. PLoS ONE. 2012;7(6):e40019.

28. García-Gómez E, González-Pedrajo B, Camacho-Arroyo I. Role of sex steroid hormones in bacterial-host interactions. Biomed Res Int. 2013;2013:928290.

29. Qiu H, KuoLee R, Harris G, Chen W. High susceptibility to respiratory Acinetobacter baumannii infection in A/J mice is associated with a delay in early pulmonary recruitment of neutrophils. Microbes Infect. 2009;11(12):946-955.

30. Tsuchiya T, Nakao N, Yamamoto S, Hirai Y, Miyamoto K, Tsujibo H. NK1.1(+) cells regulate neutrophil migration in mice 
with Acinetobacter baumannii pneumonia. Microbiol Immunol. 2012;56(2):107-116.

31. Qiu H, Kuolee R, Harris G, Chen W. Role of NADPH phagocyte oxidase in host defense against acute respiratory Acinetobacter baumannii infection in mice. Infect Immun. 2009;77(3):1015-1021.

32. Rogatzki MJ, Ferguson BS, Goodwin ML, Gladden LB. Lactate is always the end product of glycolysis. Front Neurosci. 2015;9:22.

33. Tsotakos N, Phelps DS, Yengo CM, Chinchilli VM, Floros J. Single-cell analysis reveals differential regulation of the alveolar macrophage actin cytoskeleton by surfactant proteins A1 and A2: implications of sex and aging. Biol Sex Differ. 2016;7:18.

34. Marczell I, et al. 17- $\beta$-estradiol decreases neutrophil superoxide production through Rac1. Exp Clin Endocrinol Diabetes. 2016;124(10):588-592.

35. Forlow SB, Schurr JR, Kolls JK, Bagby GJ, Schwarzenberger PO, Ley K. Increased granulopoiesis through interleukin-17 and granulocyte colony-stimulating factor in leukocyte adhesion molecule-deficient mice. Blood. 2001;98(12):3309-3314

36. Furze RC, Rankin SM. Neutrophil mobilization and clearance in the bone marrow. Immunology. 2008;125(3):281-288.

37. Potluri T, Engle K, Fink AL, Vom Steeg LG, Klein SL. Sex reporting in preclinical microbiological and immunological research. mBio. 2017;8(6):e01868-17.

38. Harris G, et al. A mouse model of Acinetobacter baumannii-associated pneumonia using a clinically isolated hypervirulent strain. Antimicrob Agents Chemother. 2013;57(8):3601-3613.

39. Hood MI, Uzhachenko R, Boyd K, Skaar EP, Ivanova AV. Loss of mitochondrial protein Fus1 augments host resistance to Acinetobacter baumannii infection. Infect Immun. 2013;81(12):4461-4469.

40. Preston JA, Beagley KW, Gibson PG, Hansbro PM. Genetic background affects susceptibility in nonfatal pneumococcal bronchopneumonia. Eur Respir J. 2004;23(2):224-231.

41. Yuan WL, Shen YJ, Deng DY. Sex bias of Acinetobacter baumannii nosocomial infection. Am J Infect Control. 2018;46(8):957-958

42. Robinson DP, Lorenzo ME, Jian W, Klein SL. Elevated 17ß-estradiol protects females from influenza A virus pathogenesis by suppressing inflammatory responses. PLoS Pathog. 2011;7(7):e1002149.

43. Pinkerton KE, et al. Women and lung disease. Sex differences and global health disparities. Am J Respir Crit Care Med. 2015;192(1):11-16.

44. Raghavan D, Jain R. Increasing awareness of sex differences in airway diseases. Respirology. 2016;21(3):449-459.

45. Farha S, et al. Effects of the menstrual cycle on lung function variables in women with asthma. Am J Respir Crit Care Med. 2009;180(4):304-310.

46. Parker D, et al. Streptococcus pneumoniae DNA initiates type I interferon signaling in the respiratory tract. $m B i o$. 2011;2(3):e00016-e00011.

47. Parker D, Prince A. Staphylococcus aureus induces type I IFN signaling in dendritic cells via TLR9. J Immunol. 2012;189(8):4040-4046.

48. Pires S, Parker D. IL-1 $\beta$ activation in response to Staphylococcus aureus lung infection requires inflammasome-dependent and independent mechanisms. Eur J Immunol. 2018;48(10):1707-1716.

49. Ahn D, et al. Acquired resistance to innate immune clearance promotes Klebsiella pneumoniae ST258 pulmonary infection. JCI Insight. 2016;1(17):e89704.

50. Rigby KM, DeLeo FR. Neutrophils in innate host defense against Staphylococcus aureus infections. Semin Immunopathol. 2012;34(2):237-259.

51. Robinson KM, et al. Influenza A virus exacerbates Staphylococcus aureus pneumonia in mice by attenuating antimicrobial peptide production. J Infect Dis. 2014;209(6):865-875.

52. Hou Y, et al. Estrogen regulates iron homeostasis through governing hepatic hepcidin expression via an estrogen response element. Gene. 2012;511(2):398-403.

53. Ikeda Y, et al. Estrogen regulates hepcidin expression via GPR30-BMP6-dependent signaling in hepatocytes. PLoS One. 2012;7(7):e40465.

54. Yang Q, Jian J, Katz S, Abramson SB, Huang X. 17ß-Estradiol inhibits iron hormone hepcidin through an estrogen responsive element half-site. Endocrinology. 2012;153(7):3170-3178.

55. Wang Y, Cela E, Gagnon S, Sweezey NB. Estrogen aggravates inflammation in Pseudomonas aeruginosa pneumonia in cystic fibrosis mice. Respir Res. 2010;11:166.

56. Singh PK, Parsek MR, Greenberg EP, Welsh MJ. A component of innate immunity prevents bacterial biofilm development. Nature. 2002;417(6888):552-555.

57. Mortensen BL, Skaar EP. The contribution of nutrient metal acquisition and metabolism to Acinetobacter baumannii survival within the host. Front Cell Infect Microbiol. 2013;3:95.

58. Mahdi L, et al. Treatment strategy by lactoperoxidase and lactoferrin combination: immunomodulatory and antibacterial activity against multidrug-resistant Acinetobacter baumannii. Microb Pathog. 2018;114:147-152.

59. Zmijewski JW, et al. Mitochondrial respiratory complex I regulates neutrophil activation and severity of lung injury. Am J Respir Crit Care Med. 2008;178(2):168-179.

60. Callahan BJ, McMurdie PJ, Rosen MJ, Han AW, Johnson AJ, Holmes SP. DADA2: high-resolution sample inference from Illumina amplicon data. Nat Methods. 2016;13(7):581-583.

61. Quast C, et al. The SILVA ribosomal RNA gene database project: improved data processing and web-based tools. Nucleic Acids Res. 2013;41(Database issue):D590-D596.

62. Parks DH, Tyson GW, Hugenholtz P, Beiko RG. STAMP: statistical analysis of taxonomic and functional profiles. Bioinformatics. 2014;30(21):3123-3124. 